\title{
State of art on efficient document co-editing in cloud collaboration
}

\author{
Tanuja Kumari Sharma and Hemraj Saini ${ }^{*}$ \\ Department of Computer Science and Engineering, \\ Jaypee University of Information Technology, \\ Waknaghat, Solan - 173234, India \\ Email: tanujarisktaker@gmail.com \\ Email: hemraj1977@yahoo.co.in \\ *Corresponding author
}

\begin{abstract}
Cloud collaboration is an important technique which helps distant users to share their work, information and files over the cloud at the same instant of time. The focus of this study is to remove the problem of the deadlock or long time waiting zone condition for requests edit threads, by the users in collaborative structure. The 'co-authoring' or 'co-editing' process generates a major problem in the form of writing confliction in concurrent and single user editor system in cloud collaboration process, hence multi-version approach is implementing as a solution to ensure no confliction in cloud collaboration environment. In this study cloud collaboration architecture is discussing which describes the efficient working of central cloud, remote cloud and local cloud in collaborating environment. The involvement of central cloud helps to minimise the storage space of data in the cloud environment with reduced redundancy factor.
\end{abstract}

Keywords: single user editor system; co-authoring; co-editing; multi-version; cloud collaboration; quality of service; user domain; cloud domain; central cloud.

Reference to this paper should be made as follows: Sharma, T.K. and Saini, H. (2019) 'State of art on efficient document co-editing in cloud collaboration', Int. J. Spatio-Temporal Data Science, Vol. 1, No. 1, pp.114-129.

Biographical notes: Tanuja Kumari Sharma is an MTech student in the Department of Computer Science and Engineering (CSE) at the Jaypee University of Information Technology (JUIT), Waknaghat, Solan - 173234, Himachal Pradesh, India. Her research interests include cloud computing and information security.

Hemraj Saini is currently working as an Associate Professor in the Department of CSE, Jaypee University of Information Technology, Waknaghat, India. He received his $\mathrm{PhD}$ from the Utkal University, Bhubaneswar, India in 2012. His research interests include network security, cloud computing, and data sciences. He has more than 18 years of teaching and R\&D experience. He has published around 100 research papers in journals and conferences of international repute. $\mathrm{He}$ has organised national and international conferences of national and international repute. Presently, he is providing his services as a reviewer, member of editorial boards and technical program committees. 
This paper is a revised and expanded version of a paper entitled 'State of art on efficient document co-editing in cloud collaboration', presented at International Conference on Smart Technologies in Computer and Communication (SmartTech-2017, Amity University, Jaipur, 27-29 March 2017.

\section{Introduction}

Cloud collaboration is the process where two or more collaborators work together by doing coordinates activities towards a set of common goals. This is an interactive process in which a user of independent group member shares interfaces rules, norms, and structures to create or share knowledge in order to do a collaborative task. This process involves working with people all over the world in real-time on a variety of documents or objects, by using different devices and virtual locations. The term cloud collaboration can define as a technique of sharing and co-authoring of objects and files in between authorise groups of users or cloud (Moghei et al., 2017). The object means a resource which gets share among a group of users of cloud collaboration services. In cloud collaboration process, documents and objects get upload in central cloud server which further collaboratively access by other distributed servers. Cloud collaboration technique allows the remote or local server users to upload and edit, share, documents or objects through different interfaces within the cloud (Zhang et al., 2012). Cloud is a server which accesses the requests of various users according to their service requirement. To complete this collaborative process the different interfaces of cloud domain and user domain help to upload and share users acknowledge and services to main cloud which in this study act as central cloud. The central cloud act as controlling manager which provide access to 'update interface services', and then make it possible to receive all the update services through the update interface to all the users in the cloud collaboration process. The object or document sharing between the different users and cloud in cloud collaboration greatly deal with security as well as with quality of service factors. Quality of services produces various quality features for sharing, editing, uploading services of an object in term of efficiency, consistency and security (Baskaran et al., 2014). Nowadays cloud-base systems are gaining enormous popularity due to a number of promising benefits, including ease of use in terms of deployment, administration and maintenance, high scalability as well as the flexibility to create new services. The growth of cloud-base services in cloud collaboration creates new problems for both user and resource share domain, which result as a number of challenges that need to discuss to ensure the successful adoption of a new paradigm. Besides this well known and often generate issues of privacy and security, a major problem facing by the users in cloud collaboration process service integrates with lack of quality of experience (QoE). If the experience quality level of cloud services does not fulfil the expectations of users involve in cloud collaboration process, then such users will reject that service or refuse its adoption in future. For this reason, the concept of QoE consist the potential to become one of the main guiding paradigms for managing quality in the cloud (Thilakanathan et al., 2014; Chen et al., 2012; Suzica et al., 2015). QoE acts as supportive key reason, for quality of services in cloud collaboration process (Tolosana-Calasanz et al., 2012; Kourtesis et al., 2014). 
In today's world cloud collaboration technique uses in business, education, and e-commerce sites and in other various technical fields to do task collaboratively at different locations. Cloud collaboration is not a term even it is a real-time application in which multiple users from distant locations can share, edit their files simultaneously. In real-time implementation, it is not so much easy to set up a direct link between the clouds, lots of knowledge about the communication as well as security field requires. The focus of this present work depends on the effort to remove the edit confliction problem. Practically, if more than one user is trying to edit the same document simultaneously lots of conflicts get produces. These conflicts consider as edit confliction error, updating confliction error and sharing interface confliction error. A confliction occurrence in cloud degrades the various important qualities of service factors such as efficiency, reliability, durability, security, correctness and performance factors (Brézillon et al., 2013; Swetha and Thippeswamy, 2014). Quality of service in itself is a big term, which not only ensures the workflow of the system also improves quality of trust factors for remote location users. Table 1 represents different quality aspects of services for cloud domain as well as for user domain. This concurrent editing process in cloud collaboration produces updating confliction, edits confliction, intention violation types of error which generally affect the workflow of the entire cloud collaboration process.

Table 1 The quality of services and their description

\begin{tabular}{|c|c|c|}
\hline \multicolumn{3}{|c|}{ Previously proposed quality of services $(Q O S)$ model for web services } \\
\hline & QOS attributes & Descriptions \\
\hline 1 & Cost (c) & Execution fee per request. \\
\hline 2 & Execution time (ET) & Time to perform the service functionality. \\
\hline 3 & $\begin{array}{l}\text { Latency time (LT) request } \\
\text { waiting time before execution }\end{array}$ & Request waiting time before execution \\
\hline 4 & Reliability (R) & 1_Failure rate \\
\hline 5 & Availability (A) & uptime / (uptime + downtime) \\
\hline \multirow[t]{2}{*}{6} & Efficiency (E) & task number \\
\hline & & throughput $\div \sum_{i=1}$ unit time $\times$ task number \\
\hline
\end{tabular}

All of the work in this paper describes about the co-editing phenomena of a document or object in the cloud collaborative domain. The editing errors of an object get produce in collaborative structure if the same document simultaneously access by all authorise users at the same time in cloud collaboration network. All the authorise users have equal access control over all the services, whether it is writing, sharing, uploading of the document in between clouds. The collaboration structural requires equal access to all services, due to this many conflicts produces with applications. A writing error occurs if at the same time more than one user edits the same object simultaneously, as a result, many types of text error, writing error and deadlock produce. To remove this problem in cloud collaboration a solution develops in the form of multi-version approach. This approach gives equal access to all the users for writing of same object at the same time. Hence, all the users independently access this common object on their personal system. This approach removes the deadlock or long time waiting condition due to which this technique is more approachable. One of the main advantages of this work is to reduce the data storage space 
in the cloud and this also resolve long waiting time problem of a common object in the 'co-editing', process of cloud collaboration.

The quality of services and their description gives in Table 1, which describe shortly about quality of service attributes such as cost, execution time, latency time, reliability, availability and efficiency type of web services factors.

Figure 1 describes the architecture of cloud collaboration which consist different type of interfaces such as editing interface, sharing interface and updating interface. These three interfaces are basic important building block of cloud collaboration model in this work.

Figure 1 Architecture of cloud collaboration (see online version for colours)

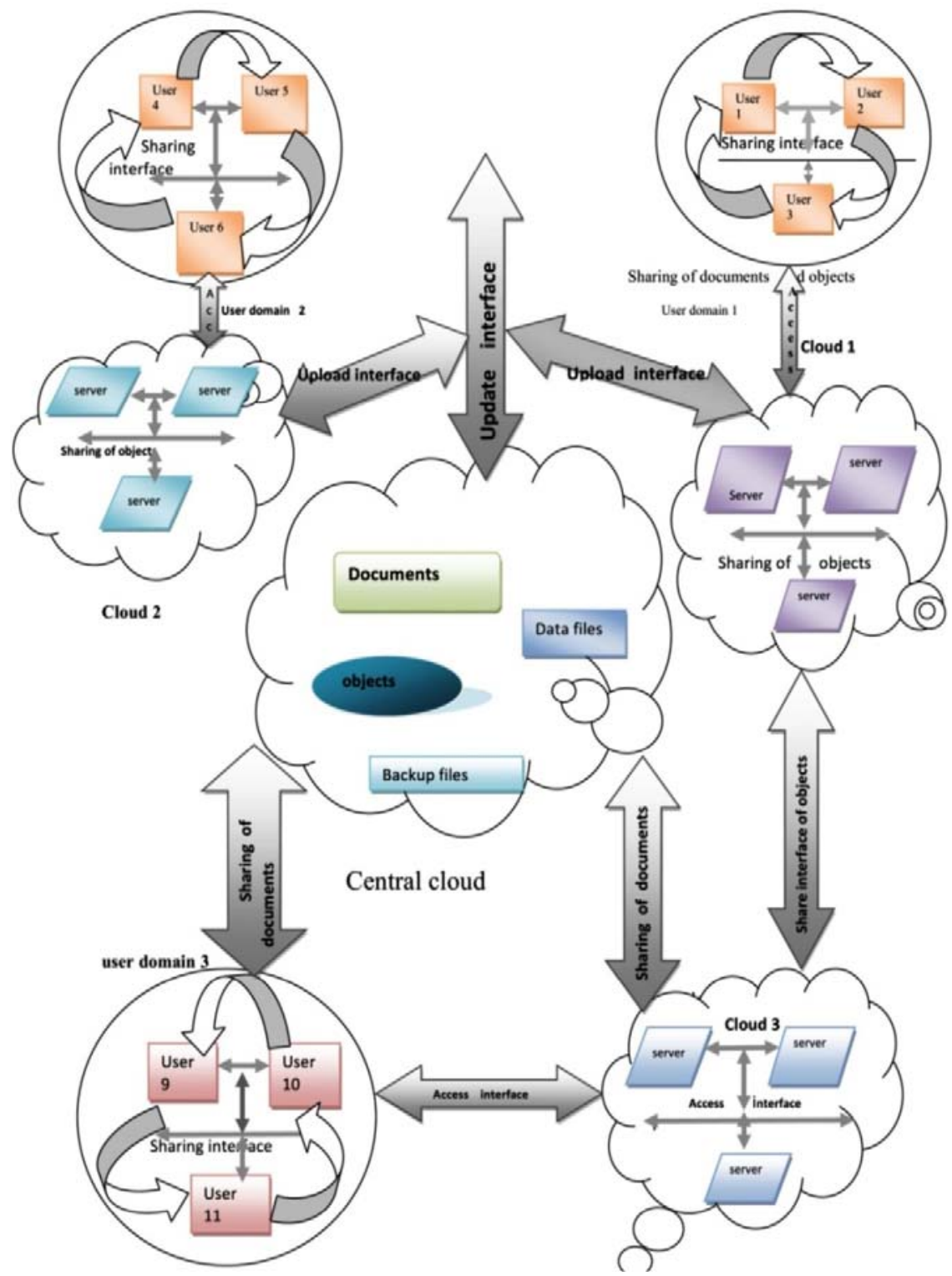




\subsection{Three important interfaces in cloud collaboration process}

\subsubsection{Editing interface}

The process of editing takes place in this architecture between local and remote cloud users. Local cloud means where users can share their services between their personal small organisations. A remote cloud means a process of sharing of resources between organisations which are indirectly linked to local cloud. As we can see in cloud collaboration architecture that cloud (domain 1) and cloud (domain 2) is local cloud if they work independently in their own domain. If cloud (domain 1) and cloud (domain 2) work in mutual file sharing environment, then it comes under remote cloud accessing of files. Hence, first of all local and remote cloud users have to registers themselves through collaboration network, after that sharing process of services take place between authorise user and cloud domain. Through the editing interface more than one user from remotely place clouds location can edit their documents collaboratively. The editing of an object is important in local cloud domain also as it is important for remote clouds. In editing interface, the documents contain various objects on which editing policies are deploying. An object describes in this study as text document, graphic document or multimedia document which can get access by the different editor at the parallel time in their different remote working environment of cloud collaboration.

\subsubsection{Sharing interface}

In sharing interface different users of remote cloud as well as local cloud can share their services in term of files as well as in the form of documents which mention as an object in this cloud collaboration working domain. A common document gets shared among all the users involve in cloud collaboration process. A common object means a copy of the document or an object which can get access and edit simultaneously at the same instant of time by more than one authorise users in cloud collaboration phenomena. The sharing phenomena of the files are one of the important factors in today internet cloud services. A sharing interface makes this possible to deliver the objects simultaneously into more than one cloud in cloud collaboration working environment without any complexity or problem. This overall sharing phenomena of documents are only possible within authorise users of cloud collaboration domain. One of the main factors which increase stability of the entire collaboration process is reliability of cloud service. Hence, it is important to share the objects within an authorising working domain so that the third entrusted party cannot be able to interrupt this object sharing process.

\subsubsection{Updating interface}

Updating interface in this work define as a process in which current authorise users in real-time, if make change in any part of the document or object, firstly it only accessible by the current user until the update change get finalised. The updating process once completes by the active user, and then it can share easily between rests of the inactive users for further processing. New final version of update object accepts by the entire authorised user in cloud collaboration process. Hence updating interface makes accessible all the cloud services without any complexion. All this process gets possible with the help of update interface. The sharing interface, editing interface and update interface are three main phases of cloud collaboration process. If update conflicts 
problem generates it can affect cloud collaboration workflow process in greater extent. As a result loss of important phases and services of cloud and user domain can take place. The architecture of cloud collaboration is depicted in Figure 1.

\subsubsection{Central cloud}

Central cloud is a main storage of cloud collaboration process. The recovery of lost documents is possible through central cloud. Main security process needs to follow here so that unusual activity can prevent by third party users. This security phase will remain future upcoming study of this architecture. Main advantages of this cloud collaboration can understand as a part of quality of services in term of reliability and availability of data. So if the crash or destroys of one cloud occur due to any technical reason it can recover from local cloud or from central cloud. The data does not lost at any cost which is very big advantage of this architecture. Hence, this architecture is reliable on the basis of its quality of service factors. Cloud collaboration itself deal with big data field, security field, cloud service field and document editing confliction field. This architecture communicates with all specific numbers of authorising users who are register with its services. Cloud collaboration architecture leads us better to know the editing confliction problem of a document or of an object.

\section{Literature survey}

Wu-Lee and Hwang (2013) in 'Workflow definition by cloud collaboration', proposed a model of cloud collaboration which defines the collaboration workflow definition language. It reduced the cost factor of services. The collaborated users did not require working in the same place and also human intervention not required to analyse the requirements of users. This work flow support distributed definition and concurrent revision problem. Cloud workflow definition language (CLWFDL) collaborate the services and objects in distributed environment.

Piechotta et al. (2016) in 'A secure dynamic collaboration environment in a cloud context', worked on to secure the cloud environment such that unauthorised user could not access collaborate process services. Cloud-based hierarchical access control (CHAC), used to comprising the research which extending upon support for fine-grained data access control that was (SDCD). This data access control was better for fine-grain data access control. A role-based access control (RBAC), model with (SDCD) introduced, which enables support for fine grained data access in dynamic cloud collaboration.

Xue and Orgun (2005) in 'Locking without requesting a lock: a consistency maintenance mechanism in internet-based real-time group editors', proposed the work based on locking without requesting a lock with a consistency maintenance mechanism in internet-based real-time group editors. This implementation was based on tickle version locking protocol. The post locking (POLO), system was used in single user editing system to prevent from the problem of editing conflicts. A different quality of services factors was analysed with the results.

Li et al. (2013) in, 'P2P-based locking in real-time collaborative editing systems' deployed a collaborated session as an abstract entity in which users may have concurrent access to the shared object during a session depending on their roles. Collaboration role-based access control (CRBAC) consists of an extended version of the RBAC model. 
CRBAC defines new entities to support access control in collaborative sessions. The CRBAC did not give a result with a description.

Dong et al. (2015) in, 'SECO: secure and scalable data collaboration services in cloud computing' discussed the communication cost evaluation and computation complexity on the bases of cloud collaboration environment. A secure data collaboration model (SECO) provided the secure services environment in the cloud. Security analysis proved that the data collaboration model was secure and thus realises fine grain access control and collusion resistance mechanism.

Prakasaa and Shigo (2015) in 'Global collaboration requirement analysis system in cloud computing' proposed a security for dynamic collaborations and resource sharing among multiple clouds which established collaboration agreement or standardised interfaces. The paper proposed a proxy-based multi-cloud computing framework. A proxy has an edge node-hosted software instance that a client or a cloud service provider (CSP) can delegate to carry out operations on its behalf. A proxy has worked as mediators for collaboration among services on different clouds. Experimental results secured the cloud services through some proxy algorithmic analysis.

Song et al. (2011) in, 'A general collaborative editing platform based on file locking mechanism' proposed work based on multilevel locks to control collaborative modelling session. The collaborative editing systems required lock mechanisms to make the collaboration more effective. The synchronous collaboration allows the notion of simultaneity however it demands more adequate perception elements. A multi-level locked mechanism that automatically manages locks in the modelling elements of 'UML, diagrams during a collaborative session. This proposed research work also proposed the multi-level lock mechanism during collaborative modelling sessions, and the observations from the empirical data collected.

Cherif et al. (2013) in 'Practical access control management for distributed collaborative editors' proposed work based on practical access control management for distributed collaborative editors. Flexible access control model introduced where the shared document and its authorisation policy got replicated in the local memory of each user. To deal with latency and dynamic access changes, the use of an optimistic access control technique took place in such a way that enforcement of authorisations becomes retroactive.

\section{Research gaps}

On the bases of previous results study, few research gaps get identifies which become the future aspects of research for the researcher. The very few aspects of proposed work in cloud collaboration require further analysis for better results.

Wu-Lee and Hwang (2013) in, 'Workflow definition by cloud collaboration' proposed the work based on the workflow definition of cloud collaboration process. The proposed results give better solutions to tackle with various types of user requirements on the bases of cloud collaboration workflow definition language (CLWFDL), for defining workflow in the working model of cloud collaboration. In this research work, following drawbacks were not discussed.

Drawbacks - lock mechanism deployment in single editor system environment of cloud collaboration produced two major drawbacks which were as follows: 
- Occurrence of inefficiency due to long time editing of the current users on a common document in a collaborative system.

- Absence of mechanisms to prevent a user from modifying the requirement of other user.

- Intervention problem still exists in cloud collaboration systems.

Xue and Orgun (2005) in 'Locking without requesting a lock: a consistency maintenance mechanism in internet-based real-time group editors' proposed the work based on locking without requesting a lock a consistency maintenance mechanism in internet-based real-time group editors. Various locks algorithms were discussed which produce good results in internet-base concurrent editing systems.

Drawbacks - multi-version mechanism of an object in a collaboration process already discussed on base of a post locking system, but not discussed how the multi-version mechanism in cloud collaboration environment work. How these multi-version approaches of an object work with the object in a single editor system in cloud collaboration environment which produces a future work for analysis as a researcher?

The various research gaps get identifies on the bases of previous study of cloud collaboration, which if analysing with good efforts may leads to more better results.

\subsection{Identification of important research gap based on previous point}

Figure 2 explains about the editing confliction problem that produces an 'updating intension problem', of an object or a document during its editing process by users in cloud collaboration environment. The object 1(o1) in Figure 2 is in editing conflict state. The object $1(\mathrm{o} 1)$ here consider as the common state of an original object where it edits by three other users that is by user 1, user 2, user 3 simultaneously. For example in present state the active user 1 do editing of the object $1(\mathrm{o} 1)$ in single user editing system, hence no one is allowing to edit the same state of an object $1(\mathrm{o} 1)$ of a document at same time.

The user 2, user 3 is in long time waiting state to edit this object 1(o1) because of busy state of a user 1 , hence this produces a deadlock in editing process. This deadlock is not good as efficient quality of service for cloud collaboration process. Collaboration in real term defines about to work together on common application at same instant of time. This research gaps refinement act as a good contribution for future valid results. A few important points of Figure 2 are representing as follows:

- In Figure 2, updating and sharing of files, documentation and identification of objects is not possible without editing conflicts which resolve with in proper time limit.

- Central cloud maintains only such object or document which is not in confliction state.

- Working aspect of this present study is relating to removal of single user common document editing confliction problem in cloud collaboration environment through multi-version approach. A lock mechanism prevents the concurrent editing conflicts of an object by change its concurrent editing process in to 'single user editor system'. This locking mechanism again produce inefficiency problem due to long time editing of a common object in 'single user editor system', of a cloud collaborative process. 
Hence inefficiency is other important aspect to remove in cloud services, otherwise this may affect various users' trustworthy services.

- The representation of a single user 'editing conflicting problem' produced on object 1 state.

- Sharing of objects between clouds in cloud collaboration process possible through object sharing interface.

Figure 2 Single user editing system confliction problems in cloud collaboration (see online version for colours)

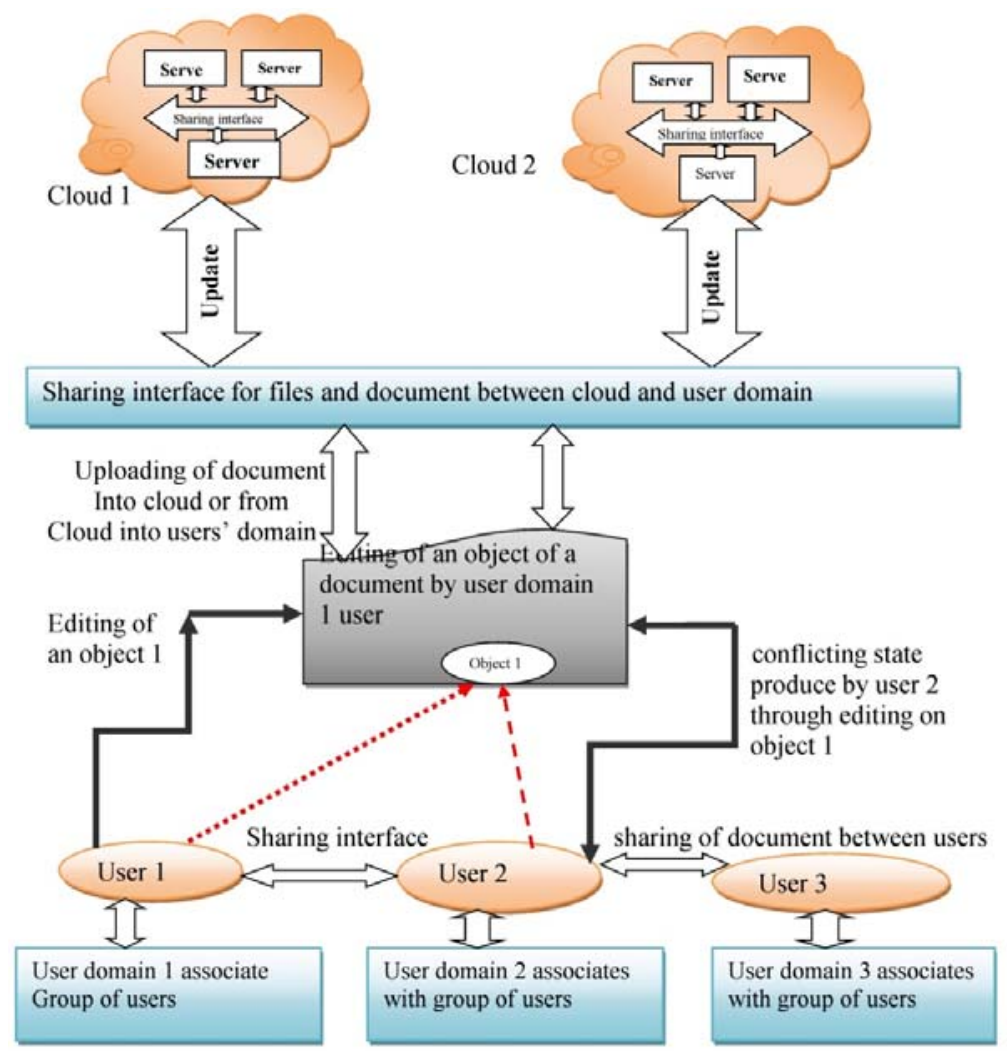

\subsection{Proposed theoretical solution for single user editing confliction problem in cloud collaboration environment}

In cloud collaboration concurrent editing error produce which affects the efficiency of collaboration in greater extent in term of quality of service. When multiple users are trying to edit the same event of the object of a document, editing confliction problem occur which cause many real life problems in cloud collaboration editing system. The solution for the editing of a common object sate in 'single user editor system', in cloud collaboration process base on the technique of multi-version of an object. The editing conflicts error generates on a common event of an object at same instant of time, which resolves through the multi-version process where more than one user edits the same state 
of an object in 'single editor system'. A present state of the user remains as it only its versions are accessing through other authorises user of cloud collaboration process. Efficiency of the system increase in 'single user edit system', because editing conflict get resolve here without requesting a lock for object editing process. The temporal storage provides for update object in local cloud or in remote cloud hence no need of extra storage space require in central cloud unless update object finalises by it (Qi et al., 2012). The central cloud only retains the final accepted copy of a document by all the authorised user of cloud collaboration process hence redundancy factor do not affects the cloud collaboration process in term of quality of services.

Figure 3 Object sharing phenomena between clouds and users domain in cloud collaboration process (see online version for colours)

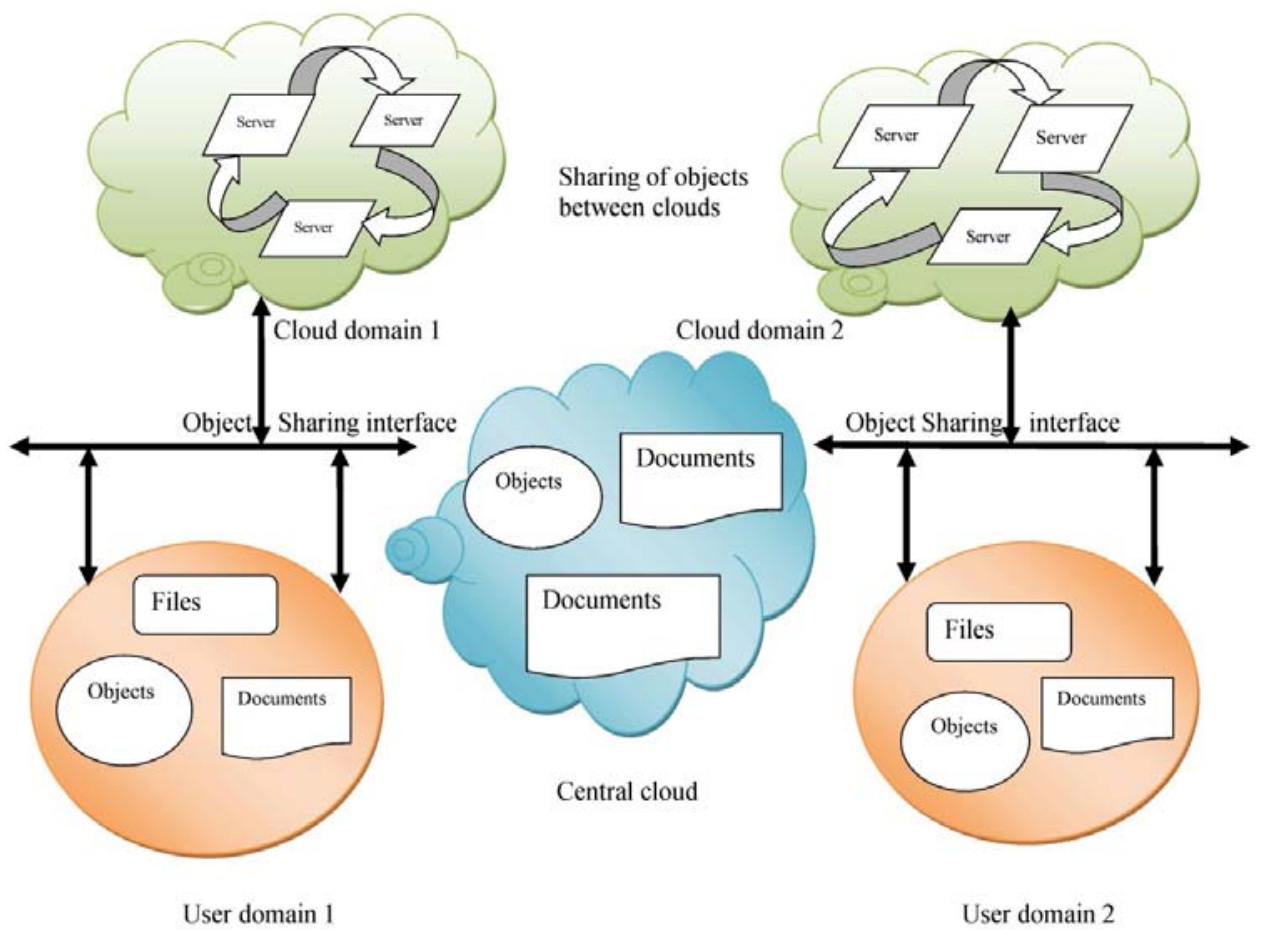

Figure 3 explains about sharing of object, document, files between clouds domain and user domain. In this figure we get to know that sharing interface is an important factor of cloud collaboration because through this interface it becomes possible to put the entire update file in central cloud. If sharing interface produce complexity then working environment of cloud user in cloud collaboration can interrupt in greater extent which may crash the all information threads permanently. The object is a resource which after updating process stores in central cloud, from where it can access collaboratively by other authorises users of cloud collaboration process. Sharing interface is active among user domain, cloud domain or between central clouds and sub domain cloud as represents in Figure 3, here in this context.

A state transition diagram of an objects states in cloud domain 
- Active state: current editing phase of an object.

- Inactive state: non editing state of an object.

- Negotiating state: negotiating state is also known as multi-version developing state.

Figure 4 Assignment of multi-version state after active state (see online version for colours)

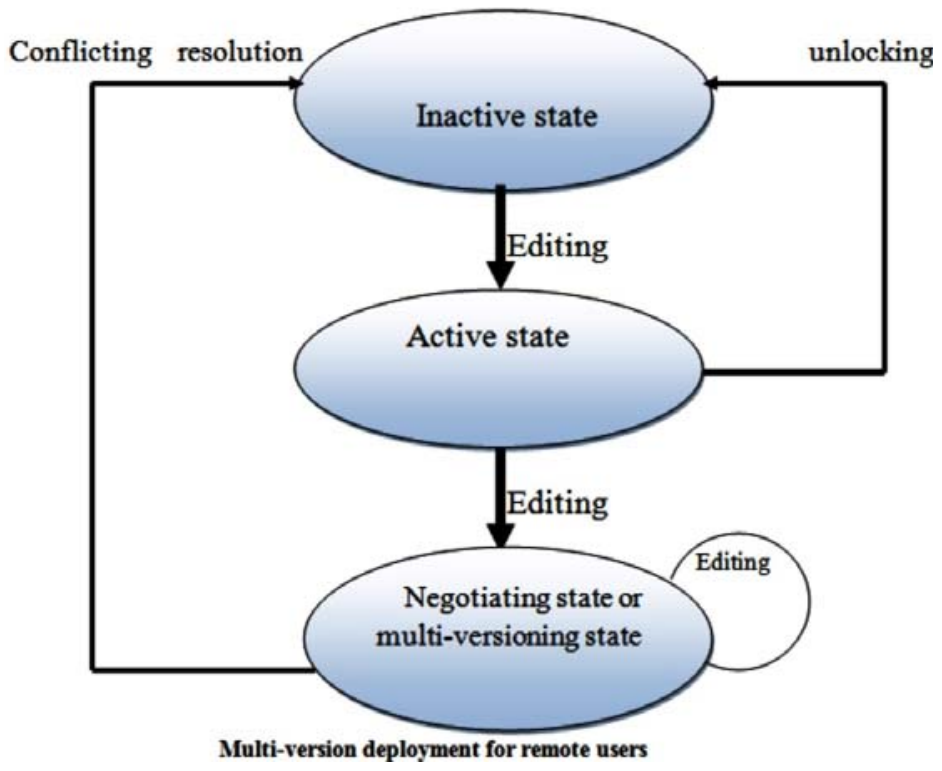

The multi-version technique is initialising with the negotiating state of a common object. If conflicts are not producing in the system, then multi-version scheme is not necessary to implement. The multi-version scheme is only become important to introduce in this work because 'single user editor system', causes the problem of inefficiency. When more than one user is trying to access the same event but not able to access it due to long time locking on original state of a common object hence editing confliction error occurs. This error can resolve through the creation of more than one version of an active object for common event editing process. Figure 4 describes about the working of a multi-version process of an object or a document. Here inactive state describe as non editing state of an object, it get over as the active state come. An active state is editing state of a document. The multi-version process is applying on negotiating state or on active state of a conflicting object to resolve the 'single user editor system', 'editing confliction problem'. If deployment of multi-version process executes the user threads appropriately then, it resolves editing deadlock problem because a same copy of document is generating on independent system of users, after which individual user can do the editing of common that is (o1), at a same time independently on their personal working systems.

\subsection{Importance of multi-version technique to resolve the editing conflicts in cloud collaboration environment}

The oval shape object which highlights in double line in Figure 5, as object $1(\mathrm{O} 1)$ which represents the real state 'editing confliction problem', inside the main document name as 
(document one) here. The main object is depicts as (document one) in which object I (O1) is defining sub content of the document 1. A multi-version approach is directive state in term of conflict resolver. This improves the efficiency because at the same time more than one user can edit the same object simultaneously. The multi-version technique gives solution for the problem of single user editor system in term of editing conflict resolver. The new version of current object is only accessible by the specific number of authorised users at a time to complete the process of 'co-authoring'. This technique also produce problem in term of update conflicts errors. For example if (user A), does the colour filling phenomena for an object (O1) then (user B), also work on the colour filling phenomena for the same object (O1) this produces conflicts in term of updating base priority, so a version control protocol implements to resolve the problem of updating intension in cloud collaboration document 'co-editing', environment.

Figure 5 Editing conflicts on circle shape object because more than one user are trying to edit this object at same time in cloud collaboration process (see online version for colours)

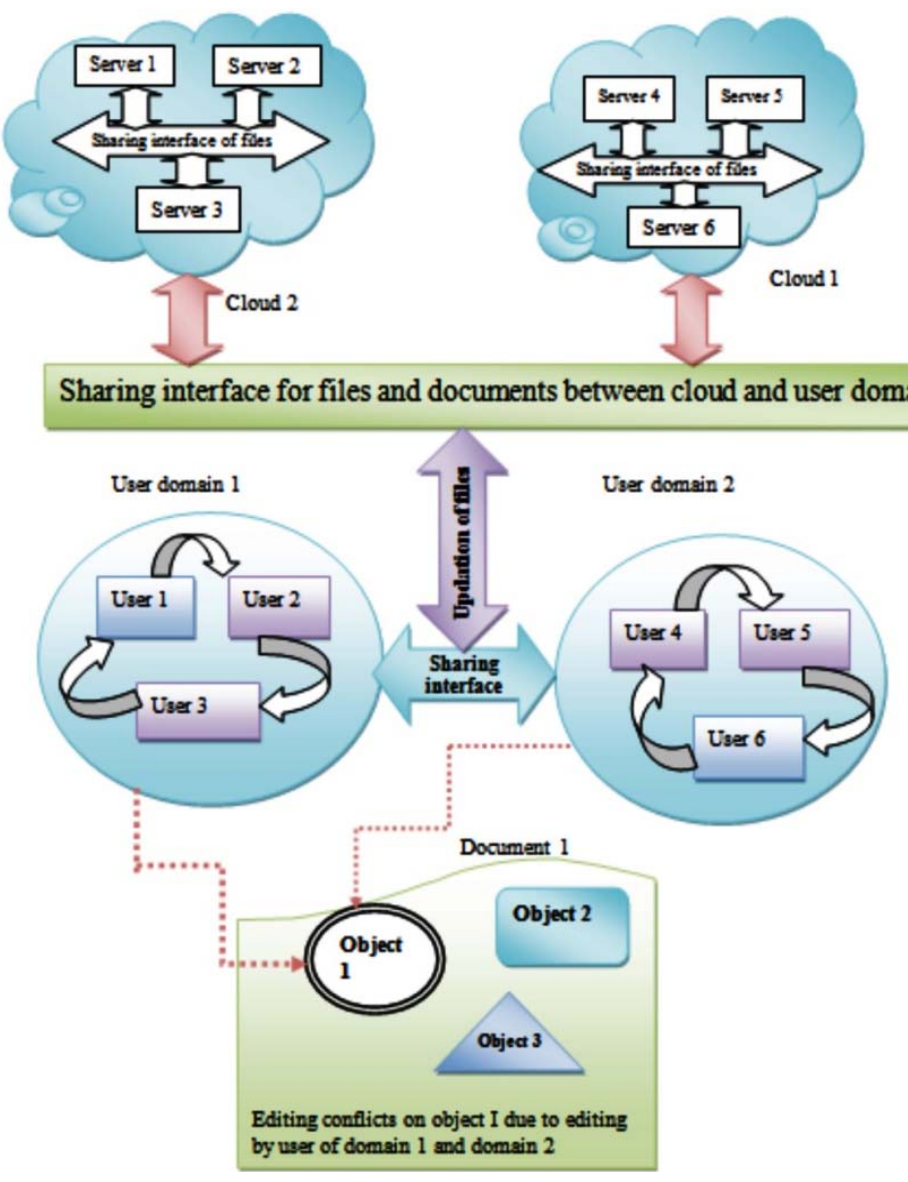




\subsection{Possible implementation methodology for multi-version process in cloud collaboration}

The deployment of multi-version process on object 1 (o1) is represented in Figure 6. In Figure 6, object 1(o1) is in busy state or in active state. When the (o1) object receives a request from waiting user token, it instantly come under multi-version process phase and resolve the waiting user token thread permanently. The multi-version process forms the similar copy of an active state of an object and delivers it to all the requests user threads. The multi-version technique does not affect the storage of cloud collaboration process, because each user threads work independently on their system. A central cloud works only with single original update version of a document after which this original document delivers simultaneously to all the users in cloud collaboration process. More than one version of object $1(\mathrm{o} 1)$ is here object (1.a) and object (1.b). Storage of these multi-version objects is temporary, until it accepts by the central cloud, because it does not use extra storage space in cloud database system. Hence this will increase reliability factor of services in cloud. The storage in local or remote cloud phase considers temporary because in central cloud only one object after update process is acceptable.

Figure 6 Deployment of a multi-version process on object 1 to resolve the problem of editing confliction in single editor system (see online version for colours)

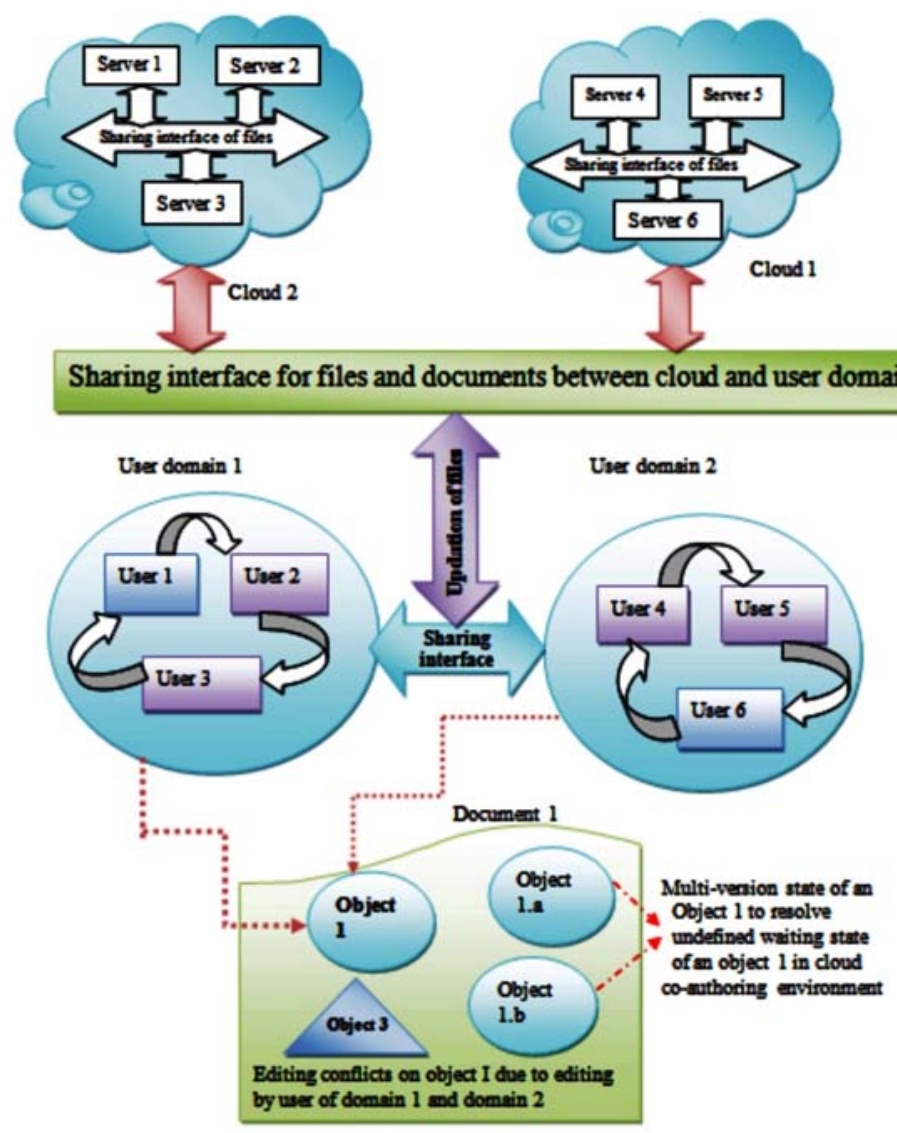




\subsection{Implementation of multi-version technique on object I state}

The version control policy of a common object 1(o1), produces control on the version of same objects for updating into the central cloud. Different states of object 1(o1) become accessible due to deployment of multi-version technique on it. Hence, a common document executes at same instant of time by authorising user over different server due to multi-version technique of a common object $1(\mathrm{o} 1)$, in cloud collaboration environment. In multi-version process temporary data storage space provides in remote or local cloud, until the final update version of original object accepts by the central cloud. The overall phenomena represents in architecture of cloud collaboration. The central cloud only accepts original update version of object, due to which storage space for cloud data in its database get minimises. Hence less priority version of a common object 1 that is (o1), get rollback or delete from central cloud to make storage space more efficient. In multi-version process lock can share between authorise group members who requests for editing of a common object 1 that is (o1) in cloud collaboration environment. In Figure 6 deployments of a multi-version technique on object 1(o1) is using to resolve the problem of editing confliction in 'single user editor system', of cloud collaboration. This describes the multi-version approach as a solution to resolve the co-editing state of an object 1(o1). Due to long time locking on an original state of object $1(\mathrm{o} 1)$, by user 1 , other user came under ideal state hence this produce editing conflict problem at same time for other users. This is the main drawback of a cloud co-editing document. To resolve this drawback, user 1 shares its lock with its conflicting user to resolve the ideal working state of an editing of an object 1(o1). The version control mechanism of multi-version process on an object $1(\mathrm{o} 1)$ resolves this problem of co-editing of a common object. After updating of an object 1(o1), it access by all authorises user in cloud collaboration environment to complete the process of 'co-authoring', of a document. The central cloud only accepts the one version of an object among the multi-versions of an object that is (o1). This technique reduces the duplication of files and resolves the file redundancy phenomena in cloud collaboration database.

\section{Conclusions}

The editing of common document produce so many problems in fields of writing domain, updating domain as well in sharing domain of objects and files in cloud collaboration process. The multi-version of common object works as solution for editing confliction problem in 'single user editor system', in collaborative environment. As we know in cloud collaboration each user has to work in collaborative structure hence equal access control is important for each services of cloud and user domain. The immediate access will provide for common object editing at same time with the help of multi-version technique. This will provide parallel access zone to all collaboration domain users for document writing services in collaborative structure, hence one big technical problem like deadlock would vanish for long time. The multi-version technique fulfils all the quality of services such as reliability and efficiency factors. This version formation approach of an event or object require minimum storage space for all user threads in cloud, so this is valid approach in term of reduction in redundancy. This approach improves the time access for information thread and user thread by removing the 
deadlock condition for long time editing of a common object in cloud collaboration process.

\section{Future work}

Future work of this study will depend on to resolve the 'updating intention violation problem'. After the deployment of multi-version technique on a common object, we can remove a deadlock permanently but simultaneously in practical sense we have to resolve the 'updating intension violation problem', which produces due to multi-version technique. This will result as selection of a final acceptable edit object by central cloud which can further share among rest of the collaboration process users. Central cloud is important in cloud collaboration hence it is necessary to retain a good functionality or reliability aspects of an object in its cloud database storage.

\section{References}

Baskaran, S., Shenbaga, V., Thenmozhi, D. and Shanmugapriya, S. (2014) 'Resource sharing platform for collaborativecloud campus', International Conference on Computation of Power, Energy, Information and Communication of IEEE.

Brézillon, P., Blackburn, P. and Dapoigny, R. (2013) 'Modeling and using context', International and Interdisciplinary Conference, Springer.

Chen, F., Dou, R., Li, M. and Wu, H. (2012) 'Enforcing QoS in scientific workflow systems enacted over cloud infrastructures', Journal of Computer and System Sciences, Vol. 78, No. 5, pp.1300-1315.

Cherif, A., Imine, A. and Rusinowitch, M. (2013) 'Practical access control management for distributed collaborative editors', Pervasive and Mobile Computing, Vol. 15, pp.62-86, Elsevier.

Dong, X., Yu, J., Zhu, Y., Chen, Y., Luo, Y. and Li, M. (2015) 'SECO: secure and scalable data collaboration services in cloud computing', Computer and Security, Vol. 50, No. C, pp.91-105.

Kourtesis, D., Alvarez-Rodríguez, J.M. and Paraskakis, I. (2014) 'Semantic-based QoS management in cloud systems: current status and future challenges', Future Generation Computer Systems, Vol. 32, pp.307-323.

Li, J., Jia, Y., Liu, L. and Wo, T. (2013) 'CyberLiveApp: a secure sharing and migration approach for live virtual desktop applications in a cloud environment', Future Generation Computer Systems, Vol. 29, No. 1, pp.330-340.

Moghei, U., Chaturvedi, B. and Lakkadwala, P. (2017) 'Cloud secure resource sharing algorithm from object based sharable environment', 4th International Conference on Reliability, Infocom Technologies and Optimization (ICRITO) (Trends and Future Directions), 2-4 September, Noida, India, DOI: 10.1109/ICRITO.2015.7359264.

Piechotta, C., Olsen, M.G., Jensen, A.E., Coleman, J.W. and Larsen, P.G. (2016) 'Secure dynamic collaboration environment in a cloud context', Future Generation Computer Systems, Vol. 55, No. C, pp.165-175.

Prakasaa, I. and Shigo, O. (2015) 'Global collaboration requirement analysis system in cloud computing', 2015 International Conference on Soft Computing and Software Engineering, Procedia Computer Science, Vol. 62, pp.332-334.

Qi, L., Dou, W., Zhang, X. and Chen, J. (2012) 'A QoS-aware composition method supporting cross-platform service invocation in cloud environment', Journal of Computer and System Sciences, Vol. 78, No. 5, pp.1316-1329. 
Song, H., Qi, Y., Ou, Z., Hu, Y., Zhang, Z. and Ye, S. (2011) 'A general collaborative editing platform based on file locking mechanism', IEEE.

Suzica, B., Reitera, A., Reimaira, F., Venturib, D. and Kuboc, B. (2015) 'Secure data sharing and processing in heterogeneous clouds', Procedia Computer Science, Vol. 68, pp.116-126.

Swetha, D. and Thippeswamy, K. (2014) 'Quality aspects and challenges in collaboration of multi-cloud - a study', International Journal of Engineering Research \& Technology (IJERT), July, Vol. 3, No. 7, pp.41-44, ISSN: 2278-0181 [online] http://www.ijert.org.

Thilakanathan, D., Chen, S., Nepal, S. and Calvo, R.A. (2014) Secure Data Sharing in the Cloud, Springer, Berlin, Heidelberg.

Tolosana-Calasanz, R., Bañares, J.A., Congducb, P. and Rana, O.F. (2012) 'Enforcing QoS in scientific workflow systems enacted over cloud infrastructures', Journal of Computer and System Sciences, Vol. 78, No. 5, pp.1300-1315.

Wu-Lee, C. and Hwang, G-H. (2013) 'Workflow definition by cloud collaboration', International Conference Paper on Collaborating Cloud Computing: Networking, Applications and Worksharing Collaborate, IEEE.

Xue, L. and Orgun, M.A. (2005) 'Locking without requesting a lock: a consistency maintenance mechanism in internet-based real-time group editors', J. Parallel Distrib. Comput., Vol. 65, No. 7, pp.801-814.

Zhang, J., Kuc, D. and Lu, S. (2012) 'Confucius: a tool supporting collaborative scientific workflow composition’, IEEE Transactions on Services Computing, Vol. 7, No. 1, pp.2-17. 